\title{
Longitudinal Space Charge Amplifier driven by a Laser-Plasma Accelerator
}

\author{
Martin Dohlus $^{a}$, Evgeny Schneidmiller $^{a}$, Mikhail V. Yurkov ${ }^{a}$, Christoph Henning $^{a}$ and Florian \\ J. Grüner ${ }^{b}$ \\ ${ }^{a}$ Deutsches Elektronen-Synchrotron (DESY), Notkestr. 85, D-22607 Hamburg, Germany \\ ${ }^{b}$ Universität Hamburg, Institut für Experimentalphysik, Luruper Chaussee 149, and \\ Center for Free-Electron Laser Science (CFEL), Luruper Chaussee 149, \\ D-22761 Hamburg, Germany
}

\begin{abstract}
A longitudinal space charge amplifier (LSCA), operating in VUV anmd soft x-ray regime, was recently proposed. Such an amplifier consists of a few amplification cascades (focusing channel and chicane) and a short radiator undulator in the end. The amplification mechanism is broadband and robust, it is practically insensitive to energy chirp and orbit jitter. Therefore, an LSCA can be considered as an alternative to a SASE FEL in the case of using laser-plasma accelerators as drivers of light sources. In this report we study generation of VUV radiation (below $100 \mathrm{~nm}$ ) in an LSCA driven by a laser-plasma accelerator with the energy of $300 \mathrm{MeV}$.
\end{abstract}

Keywords: Space charge, micro-bunching, VUV radiation

\section{INTRODUCTION}

Laser-Plasma Accelerators (LPAs) ${ }^{1-3}$ hold promise for very compact light sources due to their ultra-high field gradients excited within the plasma by high-power lasers. Routinely, they can produce electron bunches with only few fs duration, few $10 \mathrm{pC}$ charge, with energies at the GeV-level. A possibility to use these bunches for generation of femtosecond pulses of spontaneous undulator radiation was recently demonstrated. ${ }^{4}$ There are several proposals ( $\mathrm{see}^{5}$ and references therein) to use Laser-Plasma Accelerators as drivers of Free Electron Lasers (FELs).

Recently, an alternative concept for generation of high-power vacuum ultraviolet (VUV) and X-ray radiation was proposed - a Longitudinal Space Charge Amplifier (LSCA). ${ }^{6}$ The concept is based on the predicted ${ }^{7,8}$ and observed $^{9,10}$ longitudinal space charge driven microbunching instability in linacs with bunch compressors - drivers of short wavelengths FELs. It was suggested in ${ }^{6}$ that, among other applications, using LSCA as a light source based on LPA may have some advantages over FELs. In particular, due to its broadband amplification mechanism an LSCA is practically insensitive to an energy chirp induced over the entire bunch due to longitudinal space charge. LSCA is also much less sensitive to an orbit jitter than an FEL (where the overlap between electron and photon beams must be kept over a long interaction distance).

In this paper we present detailed numerical simulations of the LSCA driven by a LPA (beam parameters, that were used in the simulations, are presented in the following table). We show that a simple and compact setup can allow one to produce powerful (GW level) radiation in UV and VUV spectral ranges.

\begin{tabular}{|l|l|}
\hline bunch charge & $40 \mathrm{pC}$ \\
\hline rms bunch length & $1.5 \mu \mathrm{m}$ \\
\hline energy & $300 \mathrm{MeV}$ \\
\hline slice energy spread & $0.6 \mathrm{MeV}$ \\
\hline normalized emittance & $0.2 \mu \mathrm{m}$ \\
\hline$\sigma_{x} \approx \sigma_{y}$ in waist & $0.5 \mu \mathrm{m}$ \\
\hline peak current & $3.2 \mathrm{kA}$ \\
\hline
\end{tabular}




\section{LONGITUDINAL MICROBUNCHING INSTABILITY}

The longitudinal microbunching instability is described by effects in the longitudinal phase space $(s, \delta)$, with $s$ the longitudinal displacement and $\delta$ the relative energy deviation. Small density modulations create longitudinal self fields, and due to longitudinal dispersion the particle positions are modified, leading to densifications for certain wavelengths. Sources of the longitudinal fields are space charge (SC) effects and coherent synchrotron radiation (CSR). The space charge interaction is mostly treated as stationary process, so that the fields can easily be calculated with Poison solvers as it is done in section 2.3. The nature of CSR is more complicated and cannot be considered without the history of the source distribution or without electromagnetic field computation by field propagation in the full volume. Nevertheless effective methods have been developed that rely on purely longitudinal wakefields or impedances: ${ }^{11}$ for CSR, these impedances do not depend on the transverse shape nor on the transverse offset of the test particle. Impedances independent on the transverse offset of the test particle, but possibly dependent on the transverse shape of the source distribution are called in this report "one dimensional" and are subject of section 2.1. The one dimensional model describes amplification in the linear regime. A three dimensional model with Poisson field calculation is used in section 2.3. It is capable to calculate macroscopic effects, SC optics, non-linear effects as the generation of higher harmonics and shot noise by "macro" particles of elementary charge. Our setups to generate and grow microbunching are essentially geometrically linear, so that CSR effects are negligible or play a minor role. Most of our investigations are done with SC fields only. The approximation to neglect CSR is verified in section 2.2.

The longitudinal SC fields for short wavelengths $\lambda \propto \sigma_{r} / \gamma$ are not offset independent, (with $\sigma_{r}$ the transverse beam dimension and $\gamma$ the relativistic factor). Therefore the one dimensional model uses a transversely averaged longitudinal field (see sub-section 2.1.2).

\subsection{One Dimensional Model}

This model uses the integral-equation method (see sub-section 2.1.3) to determine the amplification of small density modulations. It is based on offset independent impedances or wakes, but it considers transverse beam properties for SC impedances and particle dynamics. In the following description we use a simplification that decouples transverse and longitudinal motion: therefore we neglect longitudinal self fields in sections with transverse dispersion and we assume that the transport matrices of such complete sections have no coupling between transverse and longitudinal phase space. The first approximation is justified by the short length and weak deflection in our transverse sections (the chicanes), the second condition can be fulfilled by design.

First we describe concepts (transport matrix, longitudinal dispersion, longitudinal impedance), than we define the gain function and refer to a simplified integral-equation method so that we can calculate effects as longitudinal oscillations, and the amplification in a single stage. Finally we consider the macroscopic bunch-lengthening and the build-up of length-energy correlation in a multi-stage scheme and compute the gain in the core of the bunch.

\subsubsection{Longitudinal Phase Space and Transport Matrix}

The equation of motion for the longitudinal phase space coordinates $s_{\nu}, \delta_{\nu}$ of particle $\nu$ is

$$
\begin{aligned}
s_{\nu}^{\prime} & =\delta_{\nu} / \gamma^{2}-x_{\nu} / R \\
\delta_{\nu}^{\prime} & =e E_{z}\left(s_{\nu}\right) / \mathcal{E}
\end{aligned}
$$

with $E_{z}$ the longitudinal electric field, $\mathcal{E}=\gamma m_{0} c^{2}$ the energy, $R$ the local curvature radius of the trajectory and $x_{\nu}$ the transverse displacement. The independent parameter is $S$, the length along the beamline.

In linear beam optics, the transport from a plane $S$ to $B$ can be described by a transport matrix $\mathbf{R}^{B S}$. In general, this matrix is written for six dimensional phase space vectors, but we restrict our investigation to linear geometries and to setups that include sub-sections with decoupled longitudinal and transverse phase spaces. Therefore the transport in longitudinal phase space is characterized by

$$
\left[\begin{array}{l}
s \\
\delta
\end{array}\right]_{B}=\mathbf{R}^{B S}\left[\begin{array}{l}
s \\
\delta
\end{array}\right]_{S}=\left[\begin{array}{cc}
r_{55}^{B S} & r_{56}^{B S} \\
r_{65}^{B S} & r_{66}^{B S}
\end{array}\right]\left[\begin{array}{l}
s \\
\delta
\end{array}\right]_{S} .
$$


We follow the convention to write indices 5 and 6 for the longitudinal coordinates. Self fields are usually not subject of linear transport descriptions, but they are in sub-section 2.1.8.

The transport matrices of a drift of length $L$ and of a symmetric four magnet chicane are

$$
\begin{aligned}
& \mathbf{D}(L)=\left[\begin{array}{cc}
1 & L / \gamma^{2} \\
0 & 1
\end{array}\right], \\
& \mathbf{C}\left(r_{56}\right)=\left[\begin{array}{cc}
1 & r_{56} \\
0 & 1
\end{array}\right] .
\end{aligned}
$$

The value $r_{56}$ of the chicane can be much larger than "total length" divided by $\gamma^{2}$. The phase spaces (over the complete symmetric chicane) are decoupled, if all pole faces are perpendicular to the $z$ axis. For our application, we use chicanes with a total length of $14 \mathrm{~cm}$ and a length per magnet of $2 \mathrm{~cm}$. Values of $r_{56}$ between 7 and 20 $\mu \mathrm{m}$ can easily be realized.

A beam with linearly correlated energy $\mathcal{E}(s)=(1+h s) \mathcal{E}_{\text {ref }}$ can be generated from a beam without correlation by the transformation

$$
\mathbf{H}(h)=\left[\begin{array}{ll}
1 & 0 \\
h & 1
\end{array}\right] .
$$

This element might be realized by an accelerating cavity operated off-crest, or it might approximate the effect of macroscopic longitudinal space charge field on a small part of the bunch.

\subsubsection{Space Charge Impedance}

The longitudinal impedance per length $Z^{\prime}=-\bar{E}_{z} / I$ relates the transversely averaged longitudinal electric field

$$
\bar{E}_{z}(\ldots)=\int d x d y \times E_{z}(x, y, \ldots) \psi(x, y)
$$

and the beam current $I . \psi(x, y)$ is the normalized transverse density function. For round beams with Gaussian profile it is

$$
Z^{\prime}(\omega)=\frac{-i Z_{0}}{2 \pi \sigma_{r} \gamma \beta} F\left(\omega / \omega_{r}\right),
$$

with $\sigma_{r}$ the rms beam radius, $\omega_{r}=\gamma \beta c / \sigma_{r}$ and the normalized frequency dependency $F(\Omega) .{ }^{12}$ The normalized frequency dependency

$$
F(\Omega)=\frac{\Omega}{2} \int_{\Omega^{2}}^{\infty} \frac{\exp \left(\Omega^{2}-u\right)}{u} d u,
$$

is plotted Fig. 1 together with low- and high-frequency asymptotes.

It has been mentioned that the transverse dependence of SC fields in not negligible for short wavelengths, as we are interested in. To some extend the transverse averaging is justified by the fact that particles perform transverse betatron oscillations. Even in a focussing channel the transverse rms dimensions, described by beta functions and emittances, are not constant. To achieve and to keep transverse dimensions of $\sqrt{\sigma_{x} \sigma_{y}} \approx 10 \ldots 15 \mu \mathrm{m}$, we use FODO lattices with $90 \mathrm{deg}$ phase advance and $40 \ldots 60 \mathrm{~cm}$ period length. For calculations with the integral-equation method we use Eq. (8) with the round beam radius $\sigma_{r}=\left\langle\sqrt{\sigma_{x} \sigma_{y}}\right\rangle$, averaged in the transverse dimensions and versus length.

\subsubsection{Integral-Equation Method and Gain Function}

The integral-equation method has been developed to determine the gain $G$ of small density modulations for a beam that is long compared to the wavelength $\lambda$ of modulation. ${ }^{13,14} G(S)$ is the ratio of the relative modulation $\tilde{I}(S) / I(S)$ to the initial relative modulation $\tilde{I}(0) / I(0)$, with $S$ the position, $I(S)$ the coasting beam current and $\tilde{I}(S)$ the superimposed current modulation. Note, that the beam might be compressed in length by $C(S)=1 / r_{55}^{S 0}$ so that $I(S)=C(S) I(0)$, and $\lambda(S)=\lambda(0) / C(S)$ depend on the position. The integral-equation is

$$
G(B)=G_{0}(B)+\int_{0}^{B} K(B, S) G(S) d S,
$$


with $G_{0}(S)$ the gain without self effects and $K(B, S)$ a kernel function. $G_{0}$ depends on $\mathbf{R}^{S 0}$ and the initial phase space distribution. In general $\mathbf{R}$ is the $6 \mathrm{D}$ transport matrix. The kernel $K$ depends additionally on $Z^{\prime}(\omega C(S))$ and $\mathbf{R}^{B 0}$. (Therefore $K(B, S)$ depends also on $\mathbf{R}^{B S}$.) For our simplified setup, without self-interaction in chicanes and without longitudinal-transverse coupling outside of chicanes, the auxiliary functions are

$$
G_{0}(S)=\Psi_{\delta}\left(k \frac{r_{56}^{S 0}}{r_{55}^{S 0}}\right)
$$

and

$$
K(B, S)=-\frac{i k r_{56}^{B S}}{r_{55}^{B 0} r_{55}^{S 0}} \frac{I Z^{\prime}\left(k / r_{55}^{S 0}\right)}{\mathcal{E} / e} \Psi_{\delta}\left(k \frac{r_{56}^{B 0}}{r_{55}^{B 0}}-k \frac{r_{56}^{S 0}}{r_{55}^{S 0}}\right),
$$

with $\Psi_{\delta}(u)=\exp \left(-0.5\left(\sigma_{\delta} u\right)^{2}\right)$, the Fourier spectrum of the Gaussian uncorrelated energy distribution, with $\sigma_{\delta}$ the relative rms energy spread.

\subsubsection{Discrete Amplifier Stage}

In a discrete stage, the modification of particle energies $\delta_{\nu}$ in the SC channel and the modification of particle positions $s_{\nu}$ are completely decoupled. Therefore we neglect longitudinal dispersion in the drift and self forces in the chicane. The discrete stage is characterized by the length of the space charge channel $L_{s c}$, the beam radius $\sigma_{r}$, the dispersion of the chicane $r_{56}$, the length-energy correlation $h$ and the relative rms energy spread $\sigma_{\delta}$ :

$$
G=\left(1-i \frac{C r_{56}}{\mathcal{E}_{\text {ref }} / e} I_{1} k_{1} L_{s c} Z^{\prime}\left(c k_{1}\right)\right) \exp \left(-\frac{\left(C k_{1} r_{56} \sigma_{\delta}\right)^{2}}{2}\right)
$$

$C=\left(1+h r_{56}\right)^{-1}$ is the compression factor and $k_{1}=2 \pi / \lambda_{1}$ the wavenumber to the initial wavelength $\lambda_{1}$. Longitudinal space charge amplification at high energy, with discrete stages, is discussed. ${ }^{6}$

The equation can be rewritten as

$$
G=\left(1-\hat{G} H\left(\frac{\lambda_{r}}{\lambda_{1}}\right)\right) L\left(\frac{\lambda_{\delta}}{\lambda_{1}}\right),
$$

with the high pass function $H(x)=2 x F(x)$, the low pass function $L(x)=\exp \left(-x^{2} / 2\right)$,

$$
\begin{aligned}
& \hat{G}=\frac{I_{1}}{I_{A}} \frac{C r_{56} L_{s c}}{\gamma \sigma_{r}^{2}}, \\
& \lambda_{r}=2 \pi \sigma_{r} /(\gamma \beta), \\
& \lambda_{\delta}=2 \pi r_{56} \sigma_{\delta} C,
\end{aligned}
$$

and $I_{A}=4 \pi m_{0} c^{2} /\left(e Z_{0}\right) \simeq 17 k A$ the Alvfen current. The normalized high- and low-pass functions are plotted in Fig. 2.

Obviously the short-wavelength-limit is determined by $\lambda_{\delta}$ and therefore by the uncorrelated energy spread. The choice $\lambda_{\delta} \simeq \lambda_{r}$ results in the condition $r_{56} C \simeq \sigma_{r} /\left(\sigma_{\delta} \gamma\right)$ and $\hat{G}=\left(I_{1} L_{s c}\right) /\left(I_{A} \gamma^{2} \sigma_{\delta} \sigma_{r}\right)$. For our parameters $\sigma_{\delta} \gamma$ is about one, so that $r_{56} C$ is close to the transverse beam size. (Self forces of the macroscopic charge distribution cause a weak decompression, so that $C$ is between 0.5 and 1.) Supposed the approximation of negligible longitudinal dispersion in the space charge channel is valid, than $L_{s c}$ could be chosen so that $\hat{G}$ is large compared to one and the maximal gain is about $\hat{G} / 3$. A gain curve for this condition is shown in Fig. 2, right. Large gains can be achieved by cascading several stages as proposed. ${ }^{15}$

Unfortunately the condition $\hat{G} / 3 \gg 1$ needs a length $L_{s c} \gg 3 \gamma^{2} \sigma_{r} \sigma_{\delta} I_{A} / I_{1}$ which is about $1.2 \mathrm{~m}$ for our parameters. This is not short compared to the path length for a quarter of the longitudinal oscillations as they will be calculated in the following. 


\subsubsection{Longitudinal Oscillations (Cold Beam)}

The solution of the integral-equation for a cold beam (without correlated or uncorrelated energy spread) in a SC channel of constant longitudinal impedance $Z^{\prime}$ is

$$
G(B)=\cos (K B)
$$

with

$$
K=\sqrt{\frac{i k I Z^{\prime}}{\gamma^{2} \mathcal{E} / e}} .
$$

This is known as longitudinal plasma oscillation: an initial density modulation with wavelength $\lambda=2 \pi / k$ causes an energy modulation and therefore a modulation in velocity that counteracts to the density. The density modulation periodically interchanges with energy modulation. The path length for one period is

$$
S_{p}=2 \pi / K=\sqrt{\frac{I_{A}}{I} \frac{Z_{0}}{\left|Z^{\prime}\right|} \frac{\lambda \gamma^{3}}{2}},
$$

and asymptotic limit for short modulation wavelength is

$$
S_{p}=2 \pi \sigma_{r} \sqrt{\frac{I_{A}}{I} \gamma^{3}} .
$$

The period length for our peak current and energy is plotted in Fig. 3.

The gain after a SC channel of length $L_{s c}$ and a discrete chicane with $r_{56}$ is again a periodical function

$$
G\left(B>L_{s c}\right)=\cos (K B)-i \frac{r_{56}}{\mathcal{E} / e} I k \frac{\sin \left(K L_{s c}\right)}{K} Z^{\prime} \cos \left(K\left(B-L_{s c}\right)\right),
$$

with period length $S_{p}$. For $K \rightarrow 0$ and $\sigma_{\delta} \rightarrow 0$ this result is identical to Eq. (13).

\subsubsection{Amplifier Stage with Continuous SC Interaction}

For beams with correlated energy spread and stages with continuous SC interaction, the integral equation has to be solved numerically, or the gain can be estimated by the product ot the cold gain times $G_{0}\left(L_{s c}+0\right)$ as

$$
G\left(L_{s c}+0\right) \approx\left[\cos \left(K L_{s c}\right)-\gamma^{2} K r_{56} \sin \left(K L_{s c}\right)\right] \Psi_{\delta}\left(k\left(r_{56}+L / \gamma^{2}\right)\right),
$$

see Eqs. $(22,11)$. Results for a discrete and a continuous stage are compared in Fig. 3.

\subsubsection{Bunch Lengthening, Generation of Correlated Energy Spread}

The bunch lengthening together with a build up of correlated energy variation is a macroscopic effect governed by the same mechanism as the longitudinal oscillations, but without periodical behaviour. In Fig. 4 we plotted the longitudinal current profile of a bunch, at the entrance and before and after the last discrete chicane of a six-stage amplification system. The longitudinal phase space distribution, can be seen in the upper-right diagram for the same bunch locations. The lower-left diagram shows the decay of the peak current in the center of the bunch. For this calculation, micro-effects from shot-noise fluctuations have been suppressed, assuming a smooth source distribution. The initial distribution was monochromatic. 


\subsubsection{Linear Multi Stage Model with Bunch Lengthening}

For a realistic simulation of SC amplification in a multi-stage system, we include the macroscopic self-effects into the linear matrix formalism. Therefore the matrix coefficients defined in Eq. (3) and used in Eqs. $(11,12)$ are determined for the core of the bunch, close to $s=0$, in presence of bunch lengthening and generation of correlated energy spread.

We applied the integral-equation method to a cascade of six amplification stages. The parameters are described in the caption of Fig. 4 and in the next section. The diagram shows amplification curves $A=C \cdot G$, the product of compression and gain. The maximal amplification is at $\lambda \approx 270 \mathrm{~nm}$. The maximal value after the first stage is about 5, compare Fig. 3. The amplification per stage reduces with the decreasing peak current. The maximal total amplification of about 4000 is sufficient to leave the linear regime or even to reach saturation: the rms shot noise before the amplifier for a bandwidth of $\Delta f \approx c / \lambda$ is $\sqrt{e I \Delta \omega / 2} \approx 1 \mathrm{~A}$, which is larger than $I / \max (A)$. Therefore higher harmonics with wavelengths can be generated. This is subject of tracking simulations, as done in the following.

\subsection{Effects from Coherent Synchrotron Radiation}

To verify the assumption of negligible CSR effects, we compare tracking simulations without and with CSR impedance. Therefore we consider short chicanes (14 cm total length, $2 \mathrm{~cm}$ magnet length), as they are used in the three dimensional model. The model of stationary CSR impedances, as it is usually used in combination with the integral-equation method, is not applicable as the radiative interaction length is comparable to the magnet length. Therefore tracking calculations have been done with transient CSR- and constant SC-impedance, using CSRtrack. ${ }^{16}$ The result of a simulation with one million particles, of the scheme described above, is shown in Fig. 5. The increased shot noise, due to the small number of particles, causes non-linear effects and saturation even after three cascades. The essential difference between the current curves is a slightly different stretching, of about six percent, indicating a different bunch lengthening due to the slowly (macroscopic) part of the CSR field. The shape and amplitude of the microscopic currents peaks are nearly identical.

\subsection{Three Dimensional Model}

This model (SC3D) uses three dimensional particle tracking in the presence of the quasi stationary field, calculated by a Poisson solver. The particles are transformed into the averaged rest frame and are sorted into the cells of an equidistant grid. The electrostatic potential is calculated by a fast convolution of the grid-distribution with the potential of one qubic cell. ${ }^{17}$ The gradient is transformed back and the Lorentz force is calculated for each particle. Tracking is done with second order transport equations for drifts, quadrupoles and bending magnets. Therefore the setup is divided into short slices of about $1 \mathrm{~cm}$ length, and after each slice the space charge kick is calculated and updated. Bending magnets are calculated without self forces.

Our results are calculated for the following setup: 90 deg FODO lattice with $40 \mathrm{~cm}$ period length. The length of the quadrupoles is $2 \mathrm{~cm}$. The first magnetic chicane is in the middle of the second half of the third FODO period. It is composed by four rectangular magnets with pole faces perpendicular to the z-axis. The magnet lengths and spacings are $2 \mathrm{~cm}$ and $r_{56}=11 \mu \mathrm{m}$. Six identical amplifier cascades, each with three FODO periods and with chicane in the last half-period are considered.

Numerical parameters: The grid for SC calculation has a width of $10 \mathrm{~nm}$ in longitudinal and $5 \mu \mathrm{m}$ in transverse direction. The aspect ratio in rest frame is close to one. The maximal tracking step in drifts is $2 \mathrm{~cm}$, quadrupoles are calculated in 4 steps. The $40 \mathrm{pC}$ bunch is simulated with 10 million or 250 million macro particles. The macro-particle charge for 250 million particles is the elementary charge. Therefore real shot noise is considered in the bandwidth of the calculation. The initial noise in simulations with 10 million particles is five time higher so that saturation is reached after less cascades.

Particle parameters: The macro-particle distribution is initialized by a Gaussian random generator with parameters as written in the introduction, but the transverse Twiss parameters are chosen different. They are numerically optimized to achieve periodical focussing in the FODO lattice, in presence of SC defocussing. The technical problem to match the source distribution to the lattice is not subject of this investigation. 
About 60 simulations have been performed with 10 million particles for different random seeds. At the entrance and after each chicane, the rms fluctuation of the Fourier spectra of the currents is calculated. For wavelengths short compared to the bunch length, the rms spectrum at the entrance is white. The rms spectra after the chicanes, normalized to the rms spectrum at the entrance, are plotted in Fig. 6. In the linear regime (after stage 1, 2 and 3), the curves can be interpreted as amplification, similar to Fig. 4. Note that this calculation is done for a bunch with longitudinal shape, while the gain model assumes a bunch with constant peak current. After stage 4, the amplitudes for short wavelength below $200 \mathrm{~nm}$ increases much stronger than in the pervious stages. This indicates the generation of higher harmonics. After the next stage, the increase is weak because the process is in or close to saturation.

Figs. 7 summarises some results of a simulation with 250 million particles. The left density plot shows the evolution of the bunch current along the beam line, with the bunch- and linac-coordinate on the ordinate and abscissa. At $L=0 \mathrm{~m}$, the current is Gaussian and corresponds to curve 1 in the up-left plot of Fig. 4. With increasing length $L$, the peak current drops and the bunch gets wider. The short chicanes at $L=1.2 \mathrm{~m} \pm 7 \mathrm{~cm}$, $2.4 \mathrm{~m} \pm 7 \mathrm{~cm}, \ldots, 7.2 \mathrm{~m} \pm 7 \mathrm{~cm}$ appear almost discrete. After chicane 4 at $4.8 \mathrm{~m}$, fluctuations due to microbunching get obvious and it can be seen, that the amplitude of the micro structure drops in the channel to the next chicane. This is due to longitudinal oscillations as discussed in sub-section 2.1.5. The distribution after the last stage is analyzed in the other diagrams: the longitudinal phase space, up-right, can be compared with that in Fig. 4. The slowly behaviour is equivalent, but the micro-bunches in the core create a fast energy variation of nearly the same height. The spikes in the current distribution have a spacing of about $280 \mathrm{~nm}$, corresponding to the maximum in the spectrum for this wavelength, and corresponding to the maximal amplification in Fig. 4. The current peaks with amplitudes up to $7 \mathrm{kA}$ are sharp and drive the spectrum even below $100 \mathrm{~nm}$.

\section{GENERATION OF RADIATION}

Behind the last cascade a radiator undulator is installed. The energy radiated into the central cone by a single electron is $\Delta \mathcal{E}_{\text {con }} \simeq 2 \pi e^{2} A_{\mathrm{JJ}}^{2} \omega K^{2} /\left[c\left(1+K^{2}\right)\right]$. Parameters of the problem are: $\omega=2 \gamma^{2} k_{\mathrm{w}} /\left(1+K^{2}\right)$ is resonance frequency, $k_{\mathrm{w}}=2 \pi / \lambda_{\mathrm{w}}, \lambda_{\mathrm{w}}$ is undulator period, $A_{\mathrm{JJ}}=\left[J_{0}(Q)-J_{1}(Q)\right], J_{n}$ is the Bessel function, $Q=\left(K^{2} / 2\right) /\left(1+K^{2}\right), K$ is rms undulator parameter. Half width of the central cone is given by $\theta_{\text {con }}=$ $\sqrt{1+K^{2}} /\left(\gamma \sqrt{N_{\mathrm{w}}}\right)$, where $N_{\mathrm{w}}$ is number of undulator periods. Radiation within the central cone has relative spectral bandwidth $\Delta \omega / \omega \simeq 1 / N_{\mathrm{w}}$. Wavepackets emitted by different electrons are not correlated, thus radiated power of the electron bunch with current $I$ is just the radiation energy from a single electron multiplied by the electron flux $I / e$ :

$$
W_{\mathrm{incoh}} \simeq\left[\frac{4 \pi^{2} e I}{\lambda}\right]\left[\frac{K^{2} A_{\mathrm{JJ}}^{2}}{1+K^{2}}\right]
$$

Let us consider the case when electron beam is modulated at the resonance wavelength $\lambda: I(z)=I_{0}[1+$ $\left.a_{\text {in }} \cos \omega\left(z / v_{z}-t\right)\right]$ with transverse distribution of the beam current density $j(z, r)=I(z) \exp \left(-r^{2} / 2 \sigma^{2}\right) /\left(\sqrt{2 \pi} \sigma^{2}\right)$, where $\sigma$ is rms transverse size of the electron beam. Radiation power of modulated electron beam is: ${ }^{18}$

$$
W=\left[\frac{\pi a_{\mathrm{in}}^{2} I^{2} N_{\mathrm{w}}}{c}\right]\left[\frac{K^{2} A_{\mathrm{JJ}}^{2}}{2+K^{2}}\right] \times\left[\arctan \left(\frac{1}{2 N}\right)+N \ln \left(\frac{4 N^{2}}{4 N^{2}+1}\right)\right]
$$

Here $N=2 \pi \sigma^{2} /\left(\lambda L_{\mathrm{w}}\right)$ is Fresnel number, and $L_{\mathrm{w}}=N_{\mathrm{w}} \lambda_{\mathrm{w}}$ is undulator length. Radiation power from modulated electron beam (25) exceeds incoherent radiation power (24) when amplitude of modulation exceeds effective amplitude of shot noise, $a_{\mathrm{in}}>\sim 1 / \sqrt{N_{\lambda} N_{\mathrm{w}}}$, where $N_{\lambda}=I \lambda /(e c)$ is number of electrons per radiation wavelength.

As it has been shown in the previous sections, controlled LSC instability is an effective tool for enhancement of electron beam density modulations. Let us illustrate this with typical example of radiation wavelength 200 $\mathrm{nm}$ and $70 \mathrm{~nm}$. Relevant quantity for beam bunching derived from simulation data, $a_{1}=<\exp \left(-i \omega t_{k}\right)>$ is represented in Fig. 8. Solid black and red curves correspond to the radiation wavelength $200 \mathrm{~nm}$ and $70 \mathrm{~nm}$, respectively. Dashed curves represent just only shot noise modulations in the electron beam density. Thus, we 
see that LSCA provides significant enhancement of the electron beam density modulations, by about three orders of magnitude in the case under study. As a result, increase of the radiation power by six orders of magnitude is expected. Note, however, that due to the broadband mechanism of amplification in LSCA the time correlations extend typically over a single cycle, so that the formula (25) cannot be used directly for calculation of the emitted power. In fact, an ultimate enhancement of power in an LSCA (with respect to spontaneous emission case) is given by $N_{\lambda} \cdot{ }^{6}$

We proceed with numerical example and consider planar undulator with the following parameters: undulator period is $2.7 \mathrm{~cm}$, peak field is $1.2 \mathrm{~T}$ at closed undulator gap of $7 \mathrm{~mm}$, number of periods is 10 . We assume that the undulator has a tunable gap which would allow us to change wavelength by changing the gap. With the energy of electrons equal to $300 \mathrm{MeV}$ maximum wavelength is achieved at closed undulator gap and is about 200 $\mathrm{nm}$.

We illustrate properties of the radiation for two wavelengths, $200 \mathrm{~nm}$ and $70 \mathrm{~nm}$. The radiation process was simulated with the code FAST. ${ }^{19}$ For this purpose, the particles' distribution, simulated with the help of SC3D, was transferred into FAST input distribution. We should note that FAST, as well as other FEL codes, uses resonance approximation, i.e. it deals essentially with narrow-band signals. The question arises wether or not one can properly simulate a process if the input signal (density modulation) has a broad band. We can answer this question as follows ( $\mathrm{se}^{20}$ for more details): within the central cone of the undulator radiation the code produces correct results even if the incoming density modulation has a wide band (in particular, if there is only shot noise having white spectrum). The accuracy of simulations of radiation properties within the central cone is on the order of inverse number of undulator periods, i.e. about $10 \%$ in our case. Main simulation results are presented in Figs. 9 - 12. Three statistical realizations are shown, that were obtained from incoming particles' distributions, simulated with SC3D (see previous sections). Typical pulse energies at the undulator exit are around 12 and 1.2 microjoules for $200 \mathrm{~nm}$ and $70 \mathrm{~nm}$, respectively. Peak powers are in the range of $1 \mathrm{GW}$ and $100 \mathrm{MW}$ for the radiation wavelength $200 \mathrm{~nm}$ and $70 \mathrm{~nm}$, respectively.

Figure 11 shows intensity distributions in the far zone for three different shots. Radiation is well collimated. Spectrum of the radiation exhibits angular dependence as in the case of single particle radiation. Angular collimation of the radiation in the far zone would allow to reduce radiation spectrum to the bandwidth to the values of about $10 \%$ defined by the number of undulator periods (see Fig. 12). Finally, let us note that (as it was discussed above) the simulations of the radiation properties are accurate to within about $10 \%$ for the case of using angular collimation in the lower plots of Fig. 12. In this case about one third of the above mentioned power is available. In the case of no collimation, the above mentioned numbers (1 GW an $100 \mathrm{MW})$ are not very accurate but give a correct order of magnitude of the radiated power.

\section{DISCUSSION}

In this paper we have shown that the concept of the longitudinal space charge amplifier can be used in the case of LPA driven compact light source. One can obtain, in particular, GW power-level femtosecond VUV pulses with the number of photons per pulse in the range $10^{12}-10^{13}$. Extension towards shorter wavelengths might be possible with higher electron energy and/or wavelength compression. Also, a stronger transverse focusing of the electron beam should help. The main open question is still an uncertainty with the uncorrelated energy spread of bunches produced in LPAs. It is clear that the measured energy spread of the beams from LPAs is dominated by energy chirp, i.e. a correlated energy change along the bunch. There are indications ${ }^{21}$ that the uncorrelated energy spread is indeed rather small but more precise measurements are still required. As a particular method for measuring uncorrelated energy spread on can consider the measurements of the LSCA gain in a single cascade with the $R_{56}$ scan (see formula (13)). Thus, as a first step towards realization of the proposed concept one can consider an experiment with one or two LSCA cascades. After such experiment an extrapolation towards shorter wavelengths would be more safe.

\section{REFERENCES}

[1] Tajima, T. and Dawson, J. M., "Laser electron accelerator," Phys. Rev. Lett. 43, 267 (1979).

[2] Leemans, W. P. et al., "Gev electron beams from a centimetre-scale accelerator," Nature Phys. 2, 696 (2006). 
[3] Esarey, E., Schroeder, C. B., and Leemans, W. P., "Physics of laser-driven plasma-based electron accelerators," Rev. Mod. Phys. 81, 1229 (2009).

[4] Fuchs, M. et al., "Laser-driven soft-x-ray undulator source," Nature Phys. 5, 826 (2009).

[5] Maier, A. R. et al., "Demonstration scheme for a laser-plasma-driven free-electron laser," Physical Review $X$ 2, 031019 (2012).

[6] Schneidmiller, E. A. and Yurkov, M. V., "Using the longitudinal space charge instability for generation of vacuum ultraviolet and x-ray radiation," Phys. Rev. ST Accel. Beams 13, 110701 (2010).

[7] Saldin, E. L., Schneidmiller, E. A., and Yurkov, M. V., "An analytical description of longitudinal phase space distortions in magnetic bunch compressors," Nucl. Instrum. and Methods A 483, 516-520 (2002).

[8] Saldin, E. L., Schneidmiller, E. A., and Yurkov, M. V., "Longitudinal space charge-driven microbunching instability in the tesla test facility linac," Nucl. Instrum. and Methods A 528, 355 (2004).

[9] Loos, H. et al., "Observation of coherent optical transition radiation in the lcls linac," Proceedings of FEL Conference, 485 (2008).

[10] Wesch, S. et al., "Observation of coherent optical transition radiation and evidence for microbunching in magnetic chicanes," Proceedings of FEL Conference, 619 (2009).

[11] Saldin, E. L., Schneidmiller, E. A., and Yurkov, M. V., "Radiative interaction of electrons in a bunch moving in an undulator," Nucl. Instrum. and Methods A 417, 158-168 (1998).

[12] Geloni, G. A., Saldin, E. L., Schneidmiller, E. A., and Yurkov, M. V., "Longitudinal wake field for an electron beam accelerated through an ultrahigh field gradient," Nucl. Instrum. and Methods A 578, 34-46 (2007).

[13] Heifets, S., Stupakov, G., and Krinsky, S., "Coherent synchrotron radiation instability in a bunch compressor," Phys. Rev. ST Accel. Beams 5, 064401 (2002).

[14] Huang, H. and Kim, K., "Formulas for coherent synchrotron radiation microbunching in a bunch compressor chicane," Phys. Rev. ST Accel. Beams 5, 074401 (2002).

[15] Dohlus, M., Schneidmiller, E. A., and Yurkov, M. V., "Generation of attosecond soft x-ray pulses in a longitudinal space charge amplifier," Phys. Rev. ST Accel. Beams 14, 090702 (2011).

[16] Dohlus, M. and Limberg, T., "Csrtrack version 1.2 users manual," DESY (2007).

[17] Qiang, J., Lidia, S., Ryne, R., and Limborg-Deprey, C., "Erratum: Three-dimensional quasistatic model for high brightness beam dynamics simulation," Phys. Rev. ST Accel. Beams 9, 044204 (2006).

[18] Saldin, E. L., Schneidmiller, E. A., and Yurkov, M. V. Nucl. Instrum. and Methods A 539, 499 (2005).

[19] Saldin, E. L., Schneidmiller, E. A., and Yurkov, M. V., "Fast: a three-dimensional time-dependent fel simulation code," Nucl. Instrum. and Methods A 429, 233 (1999).

[20] Saldin, E. L., Schneidmiller, E. A., and Yurkov, M. V., [The Physics of Free Electron Laser], Springer, Berlin (2000).

[21] Lin, C. et al., "Long-range persistence of femtosecond modulations on laser-plasma-accelerated electron beams," Phys. Rev. Lett. 108, 094801 (2012). 


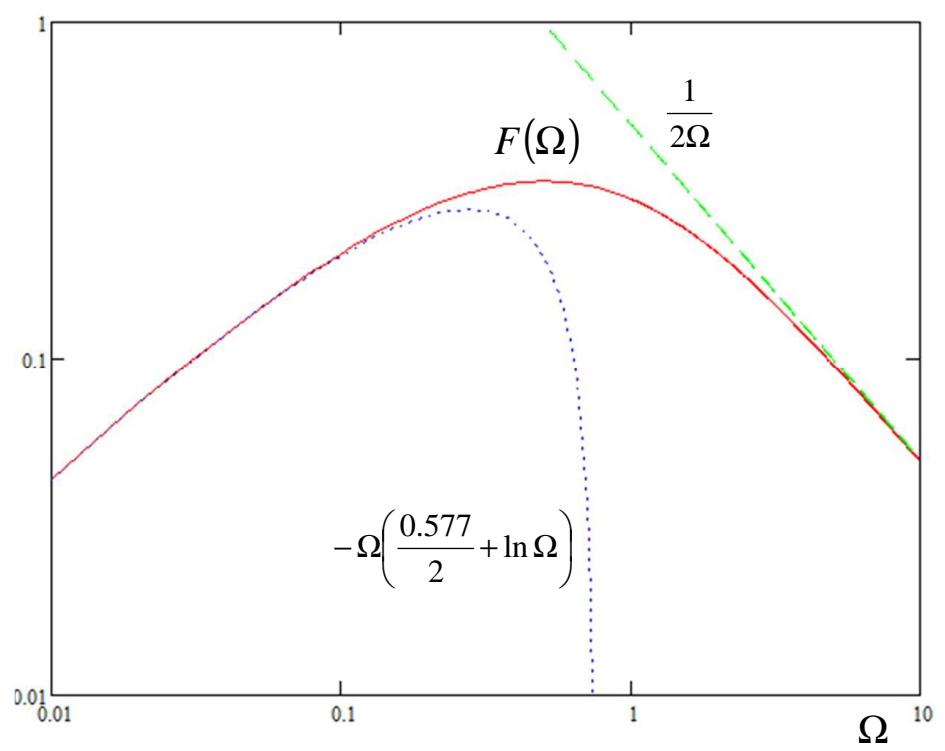

Figure 1. Normalized frequency dependency $F(\Omega)$ of the space charge impedance and its asymptotic behaviour.
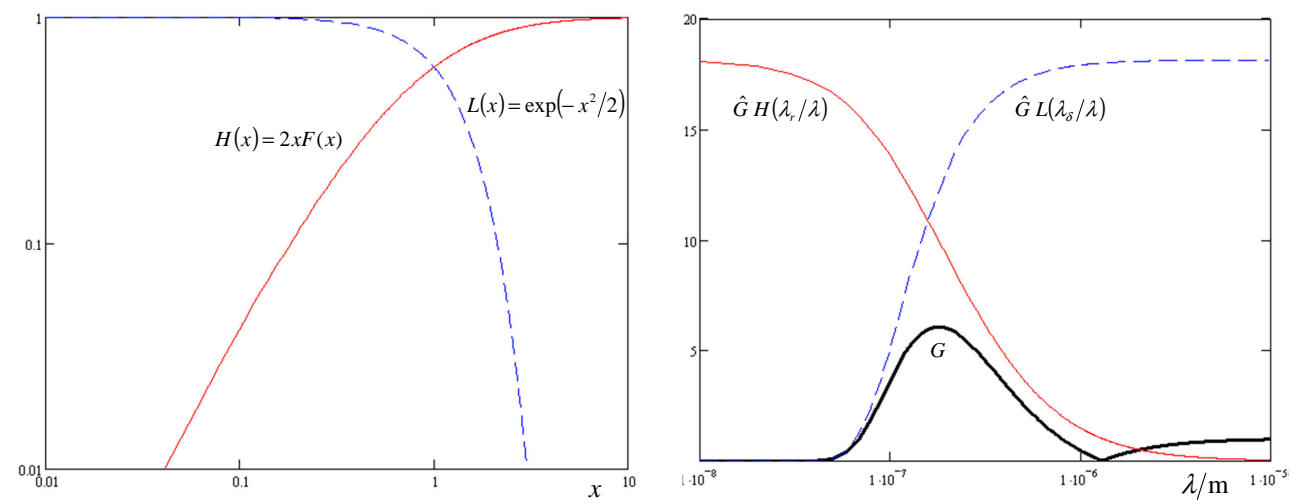

Figure 2. Left: Normalized high- and low-pass filter. Right: Gain curve of amplification stage, for $\sigma_{r}=15 \mu \mathrm{m}, S=1 \mathrm{~m}$, $C=1, r_{56}=\sigma_{r} /\left(\sigma_{\delta} \gamma\right)$, energy and current according to our parameters
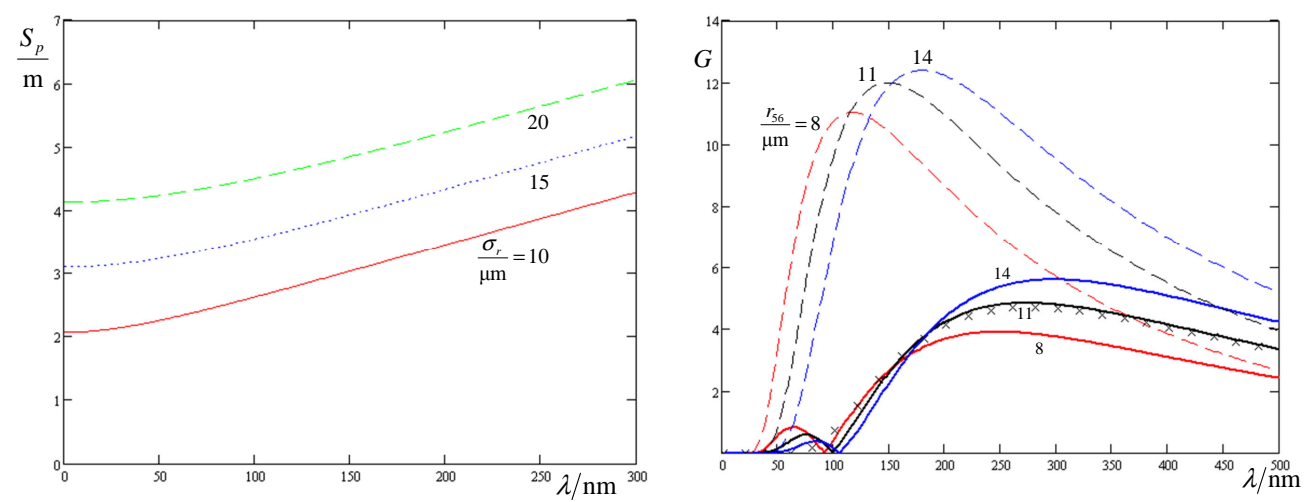

Figure 3. Left: Period length of longitudinal oscillations. Right: Dashed/solid line, gain of one stage with discrete/continuous drift. Crossed points for approximation (23). $L=1.2 \mathrm{~m}, \sigma_{r}=10 \mu \mathrm{m}$, energy and current according to our parameters 

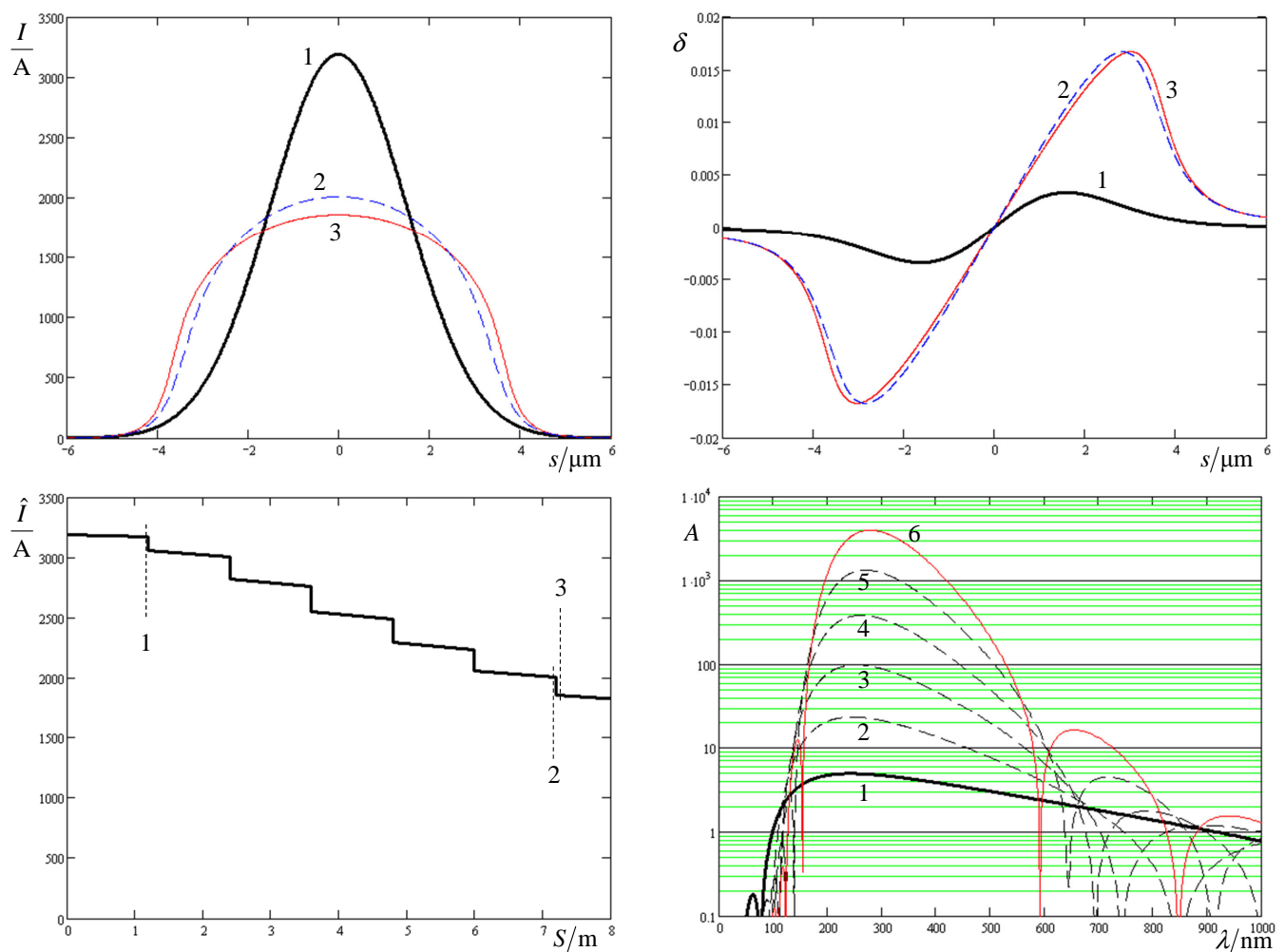

Figure 4. Up-left: bunch current vs. bunch length, up-right: longitudinal phase space. For beamline positions 1 at entrance of first chicane, 2 and 3 at entrance and exit of last chicane. Down-left: current in bunch center vs. beamline coordinate. Down-right: amplification after each of the chicane. For $L_{s c}=1.2 \mathrm{~m}, r_{56}=10.8 \mu \mathrm{m}, \sigma_{r}=10 \mu \mathrm{m}$, bunch charge and energy according to our parameters
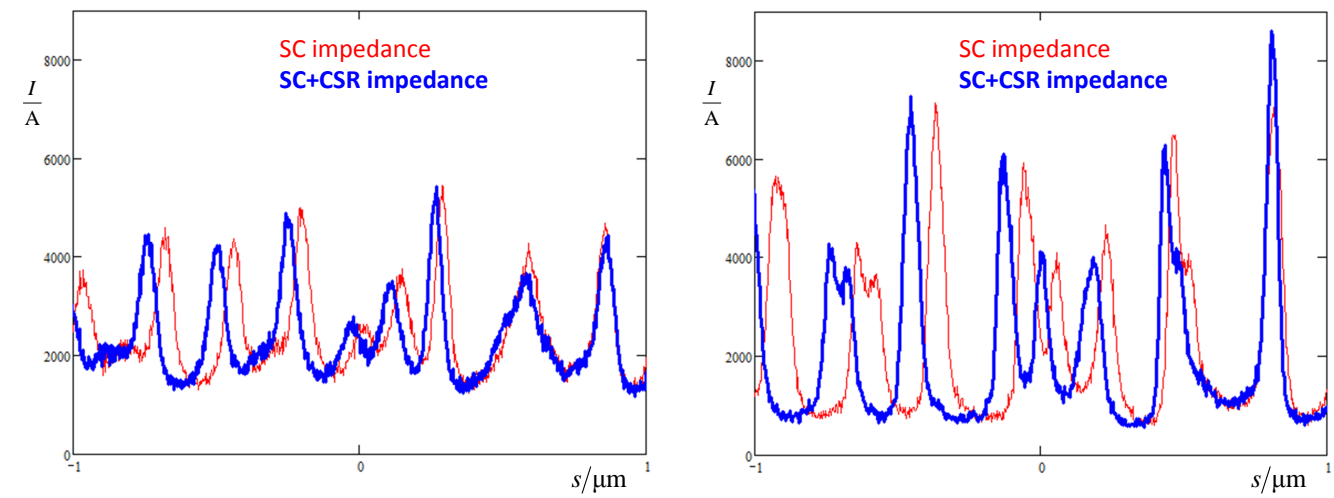

Figure 5. Bunch current with stationary SC impedance and without/with transient CSR impedance calculated by CSRtrack for one million macro particles. Left: after 3 cascades, right after 4 cascades. Parameters as in Fig. 4 , but with chicanes of $14 \mathrm{~cm}$ length 


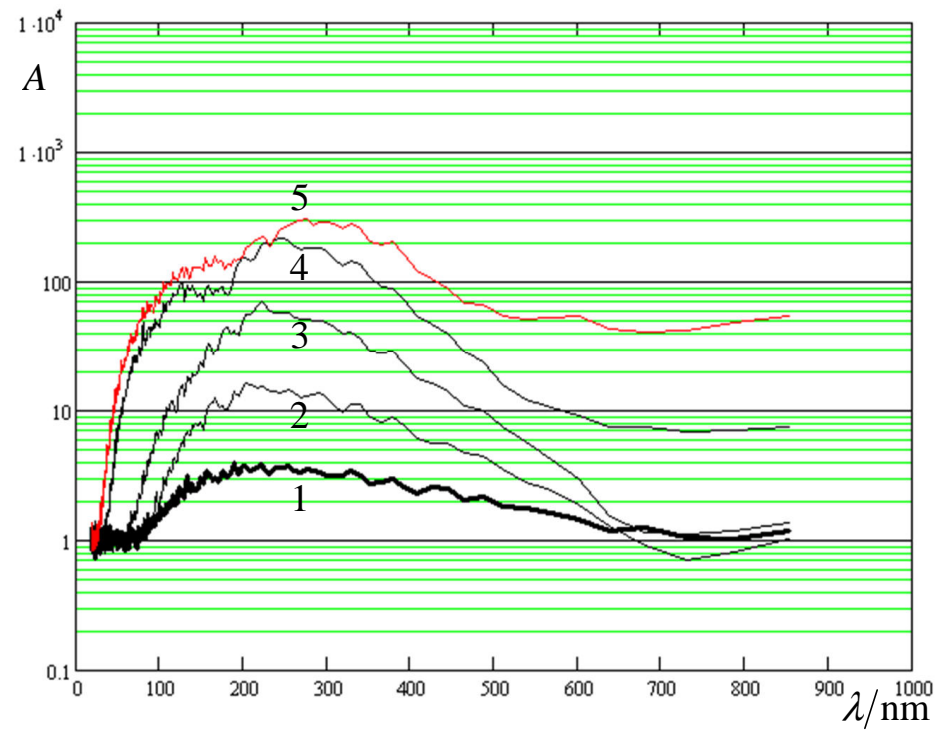

Figure 6. Simulation with 10 million particles and three dimensional space charge effects. The spectral shot noise is calculated from 60 simulations with different random seeds. The curves are the shot noise after chicanes $1 \ldots 5$, normalized to the shot noise at the entrance of the scheme. Parameters as in Fig. 4, but with chicanes of $14 \mathrm{~cm}$ length
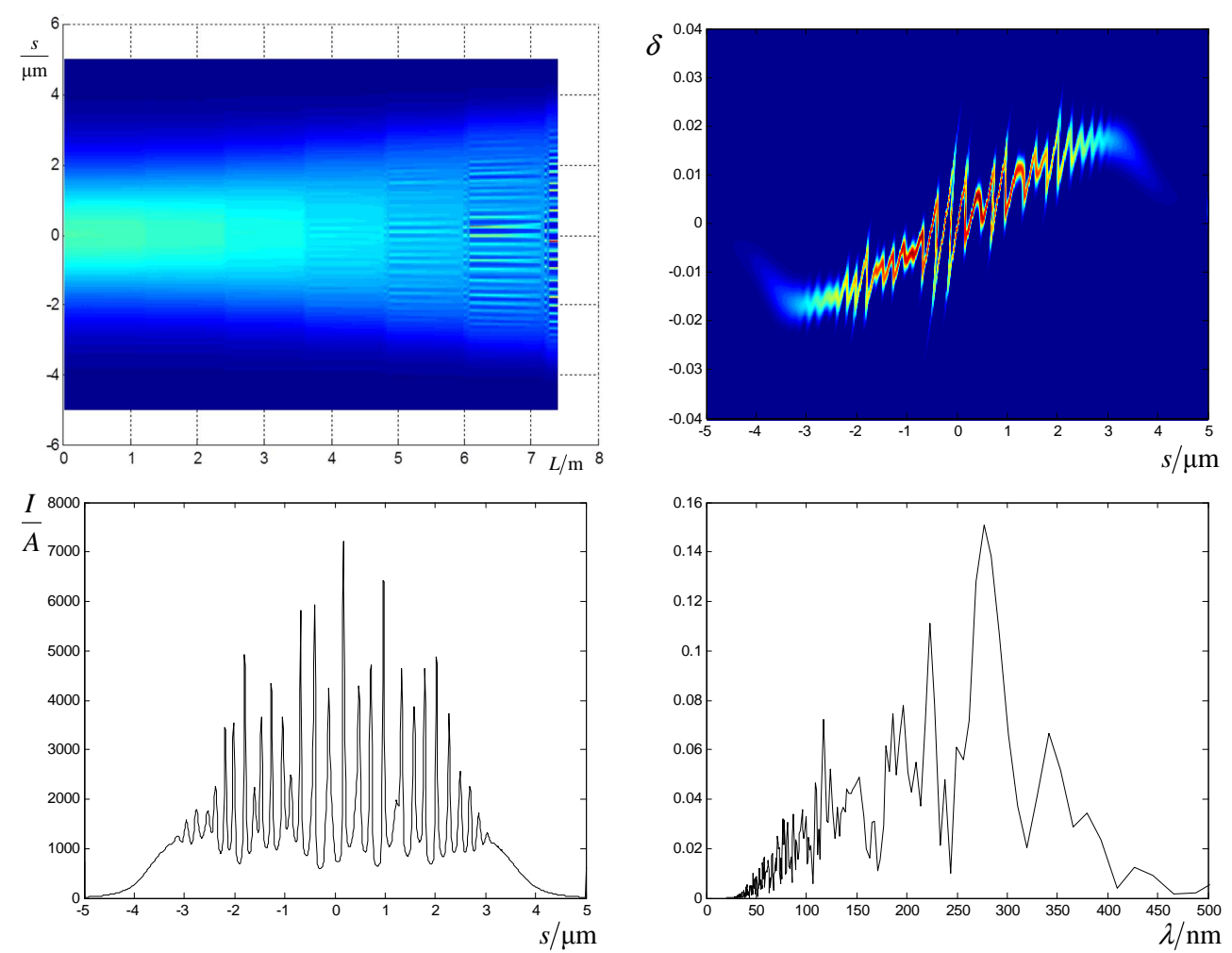

Figure 7. Simulation with 250 million particles and three dimensional space charge effects. Up-left: current profile along beam line. The vertical and horizontal axes are the bunch- and beamline-coordinates, the gray scale corresponds to the current density. Up-right: longitudinal phase space after last chicane, down-left: current profile after last chicane, right-left: current spectrum after last chicane. Parameters as in Fig. 4, but with chicanes of 14cm length 


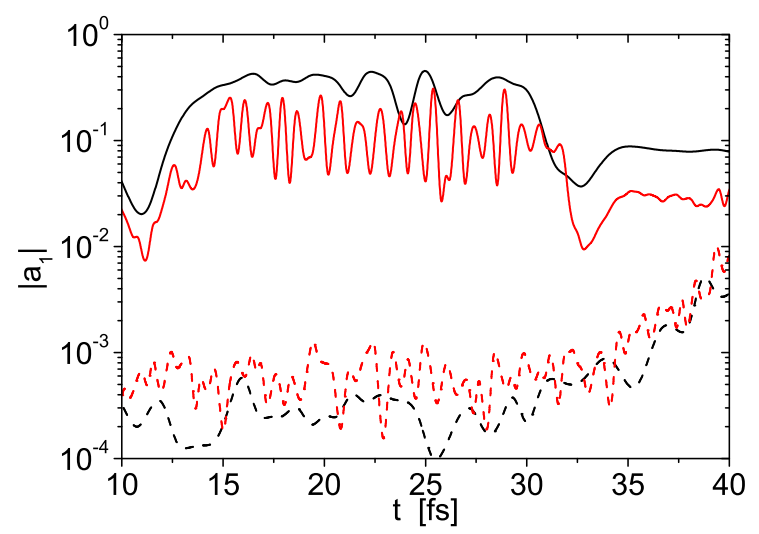

Figure 8. Beam bunching (solid curves) along the electron pulse at the undulator entrance. Black and red curves show beam bunching at the wavelengths of $200 \mathrm{~nm}$ and $70 \mathrm{~nm}$, respectively. Dashed curves show beam bunching corresponding to just only shot noise in the electron beam.
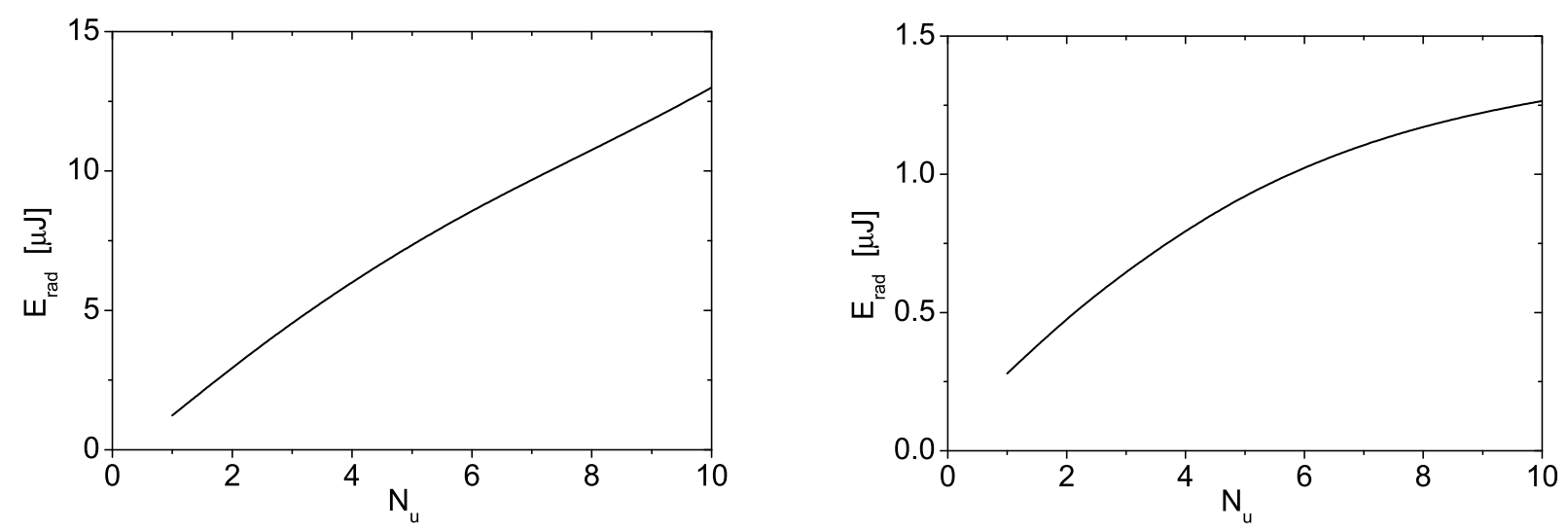

Figure 9. Energy in the radiation pulse versus undulator length. Left and right plot correspond to undulator tuning to the resonance wavelength $200 \mathrm{~nm}$ and $70 \mathrm{~nm}$, respectively. 

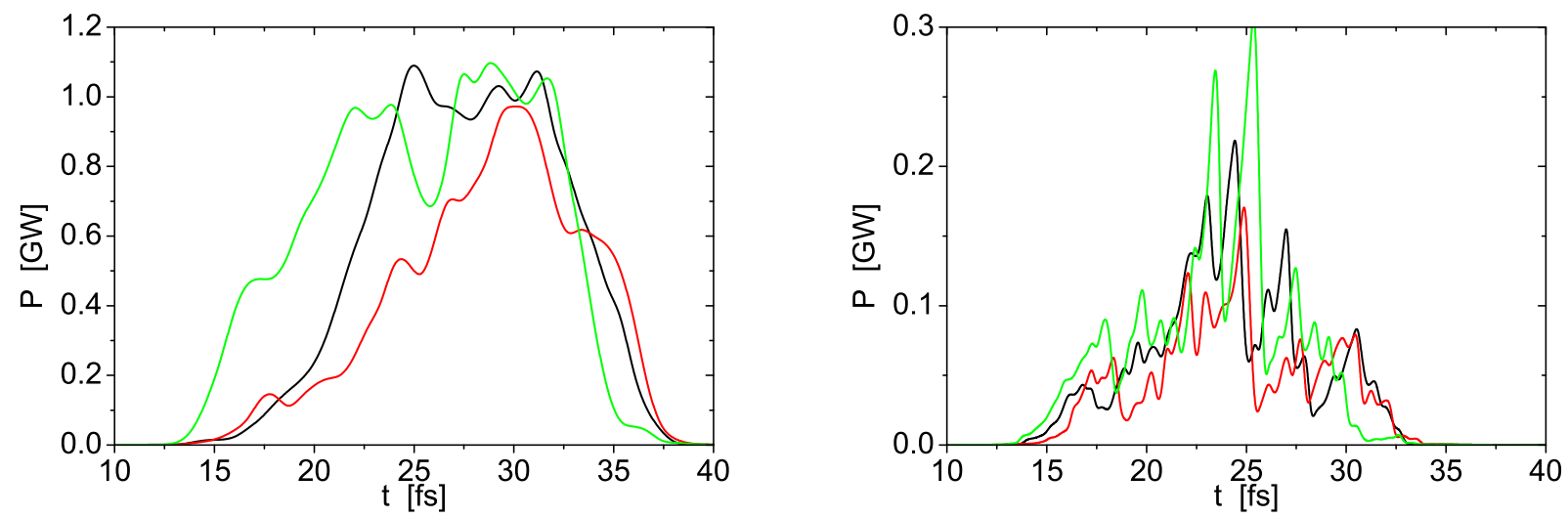

Figure 10. Temporal structure of the radiation pulse for the radiation wavelength $200 \mathrm{~nm}$ (left plot), and $70 \mathrm{~nm}$ (right plot). Three different colors correspond to three different shots.
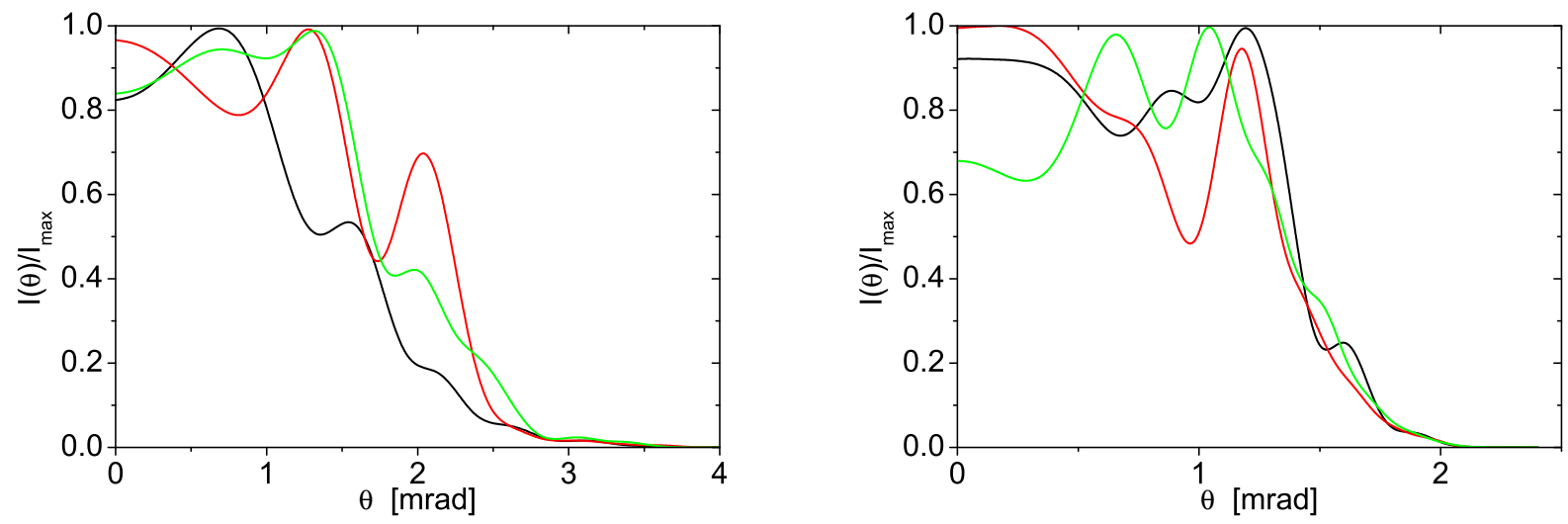

Figure 11. Intensity distribution in the far zone. for the radiation wavelength $200 \mathrm{~nm}$ (left plot), and $70 \mathrm{~nm}$ (right plot). Three different colors correspond to three different shots. 

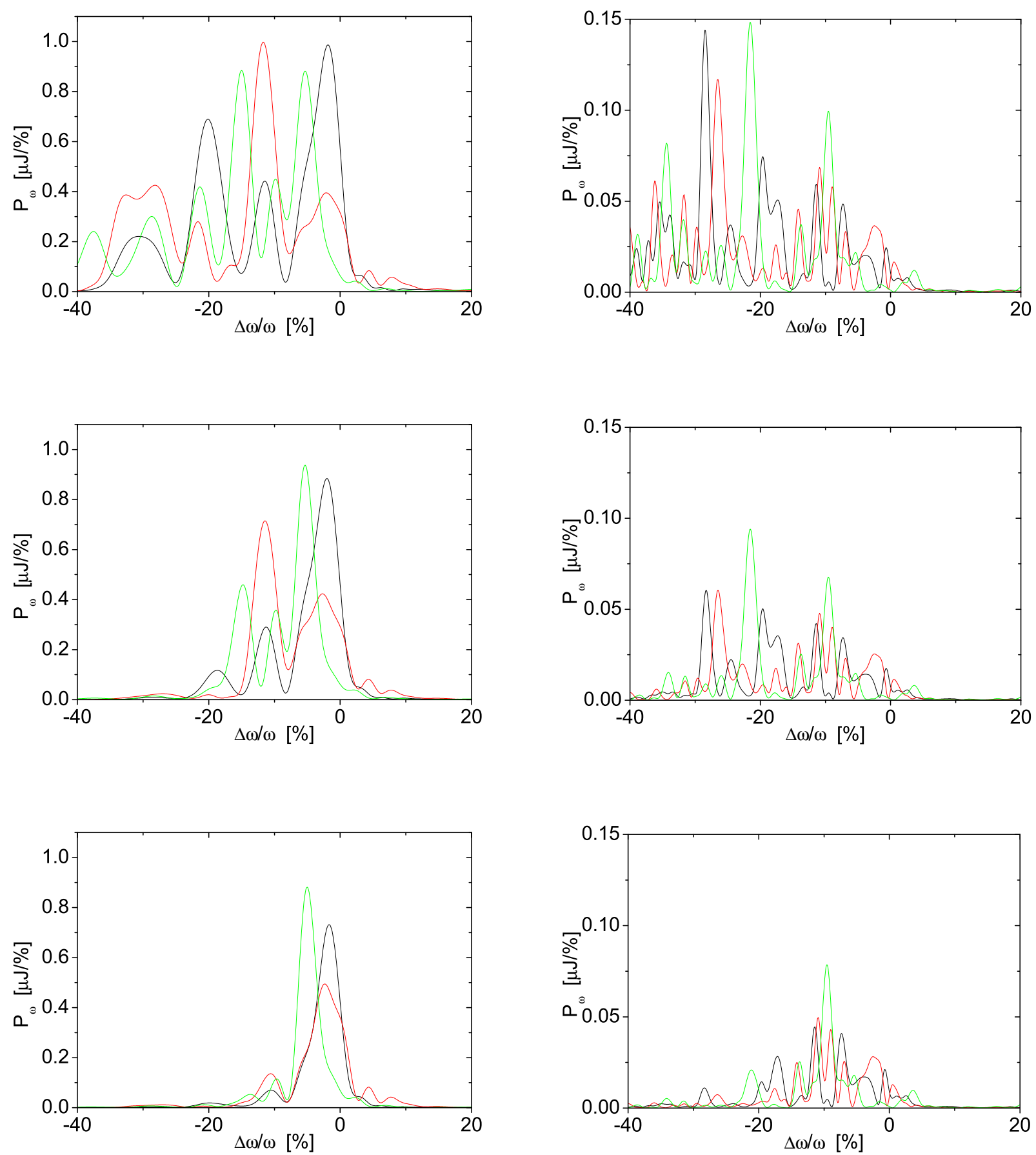

Figure 12. Spectral structure of the radiation pulse in the far zone for the radiation wavelength $200 \mathrm{~nm}$ (left column), and $70 \mathrm{~nm}$ (right column). Three different colors correspond to three different shots. Upper plots represent spectrum of the full radiation pulse. Middle plots represent spectrum of radiation pulses in the cone containing $50 \%$ of the radiation power. Lower plots represent spectrum of radiation pulses in the cone containing $30 \%$ of the radiation power. 